\title{
Direct Numerical Simulation of Forced Flow Dielectric EHD within Charge Injection Atomizers
}

\author{
John Shrimpton and Agissilaos Kourmatzis \\ Energy Technology Research Group \\ School of Engineering Sciences \\ University of Southampton \\ Southampton S017 1BJ, UK
}

\begin{abstract}
A charge injection atomizer functions by introducing electric charge discharged from a high voltage electrode into a dielectric liquid, which subsequently atomizes the ejected liquid jet. Atomizer evolution thus far has proceeded through trial and error analysis of the experimentally measured electrical characteristics of the atomizer and of the quality of atomization. Within the atomizer, a coupled space charge and electric field exist, which can alter the internal flow pattern, thus creating electrohydrodynamic (EHD) instabilities that affect atomizer operation. Such a system has not been simulated in the past under forced flow conditions. In this work we simulate the internal flow of such a charge injection device in two dimensions; using experimental based boundary conditions. Initial results indicate that in the linear injection regime defined by the experimental data, the flow is only slightly unstable but in the transitional and highly non-linear regimes, the coupled space charge and electric field produce more instability in the liquid that must be investigated further.
\end{abstract}

Index Terms - Charge injection atomizers, EHD, dielectric liquids.

\section{INTRODUCTION}

A charge injection atomizer utilizes the fact that when a dielectric liquid is subjected to a high voltage, the electrical force produced by the applied electric field and injected space charge may be used in order to produce a fine spray [1]. The development of charge injection atomizers to date has proceeded mainly via experimental analysis, in order to quantify performance in terms of electrical and spray characteristics [2-10].

Figure 1 shows a schematic of a charge injection atomizer. Fluid flows past an electrode at negative high voltage and charge is transferred to the dielectric liquid. Upon exiting the orifice, the electrical Lorentz force dependant on the charge injected and the applied electric field, acts radially, due to the repulsion of like charges. This breaks up the ejected jet into ligaments [5], and finally in to small atomized droplets.

Atomizer development has been carried out by a number of researchers including the original designs by Kim and Turnbull [8], followed by Robinson et al [9], Kelly [11-12], Yule and Shrimpton et al [13-14,16], Rigit and Shrimpton [4-5] Romat and Badri [15] as well as Al Ahmad et al [7]. The majority of this work has concentrated on 'point-plane' layouts where the point high voltage emitter electrode is typically a stainless steel needle and the plane contains the orifice. More recently, work has concentrated on different electrode shapes and multiple orifices [16], as opposed to

Manuscript received on 19 January 2010, in final form 19 April 2010. the conventional point-plane single orifice designs. Furthermore, pulsed voltage-steady flow atomization is currently being investigated by the authors as a prelude to fully pulsed voltage, pulsed flow charge injection. The operation of both the conventional and more developed designs in the steady voltage regimes is well understood, but mainly from an empirical point of view.

One process that has not been examined or understood in detail, is the actual charge injection mechanism taking place within the atomization device, although an electro-chemical process is one of the more accepted theories [17]. Even though the charge injection mechanism is not well understood, all of these atomization systems with different geometries yield extremely similar current-voltage (I-V) characteristics [2-16], indicating that the charge injection mechanism is an emitter surface phenomenon rather than a geometry dependant mechanism. The research into the electrical characteristics has also been complemented with the analysis of spray characteristics, such as the investigation of droplet size distribution [2-3,5] and charge-diameter correlations [18].

The studies above have concentrated on empirically understanding what contributes to atomization and how we can develop more efficient atomizers, both from an electrical and spray efficiency point of view. In this paper, the topic of discussion is the internal flow within a charge injection atomizer, and we concentrate on this area for the reasons to follow. 
It is necessary to understand the electrohydroydnamic (EHD) turbulence generated within charge injection atomizers as it will aid in the future design of such devices, while also revealing interesting information regarding EHD as a whole. Practically, the reason why we pursue such research is due to observations from experimental data. Steady state multiple-orifice inefficiencies have been observed [16], and it is likely that pulsed voltage and/or pulsed flow will also contribute to spray inefficiency. Furthermore, it is known that there is a Reynolds number dependence on the performance of the atomizer [19]; specifically, at high Reynolds numbers there is a decrease in the rate of increase of spray specific charge. Therefore, it is necessary to observe the nature of chaotic flow within these devices. In addition, observation of a transition to chaos is also of interest, as it will provide vital information regarding how the level of charge injection affects the chaotic flow present. This has been investigated by others [20-23], however only in highly regular domains of no practical interest. In the work presented here, we analyse the internal flow of a typical charge injection atomizer geometry for the first time, and this is done using a 2D finite volume code.

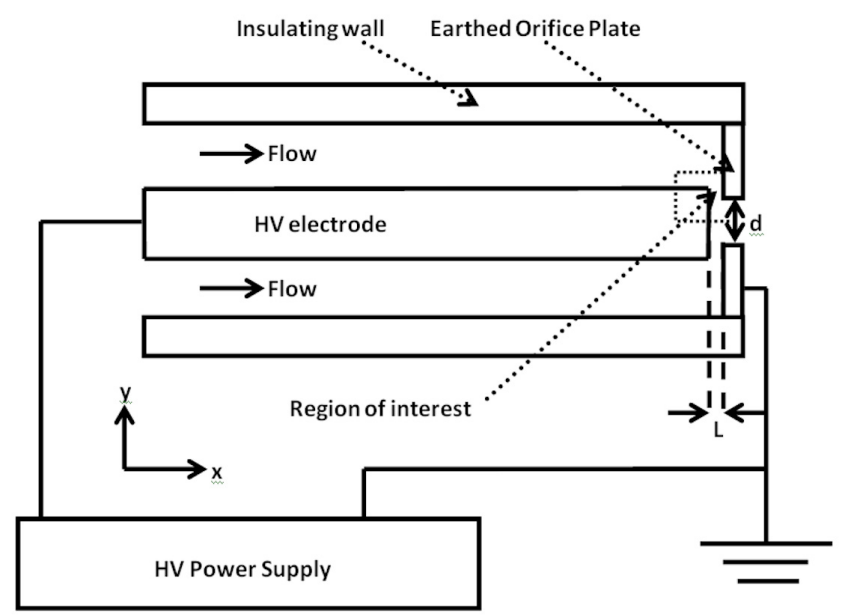

Figure 1. The charge injection atomizer concept showing the flow direction, high voltage electrode, electrode gap and simulated region of interest.

The paper shall be organized as follows: the governing equations shall be stated, followed by details on the code used. Secondly, the boundary conditions used shall be provided, along with mesh details and fluid physical properties, which shall be used to calculate relevant non-dimensional numbers. Finally, the results will be presented and discussed.

\section{GOVERNING EQUATIONS AND CODE DETAILS}

Here, the relevant governing equations of the problem are stated, followed by a brief description of the code.

\subsection{GOVERNING EQUATIONS}

In these simulations we predict the motion of an electrically charged fluid and therefore solve momentum, mass and charge conservation equations. Equations (1) to (5) [24-26] are the conservative form of the non-dimensional governing equations for the forced flow problem, assuming incompressible flow and constant density, where the velocity scale is given by $\mathrm{U}_{0}$.

$$
\begin{aligned}
& \frac{\partial}{\partial t^{*}}\left(Q^{*}\right)+\frac{\partial}{\partial x_{i}^{*}}\left(Q^{*} U_{i}^{*}\right)=\frac{1}{\operatorname{Re} S c_{E}} \frac{\partial}{\partial x_{i}}\left(D^{*} \frac{\partial Q^{*}}{\partial x_{i}^{*}}\right)- \\
& \frac{\kappa_{0} E_{0}}{U_{0}}\left(E_{i}^{*} \frac{\partial Q^{*}}{\partial x_{i}^{*}}+Q^{* 2}\right)+\left[\frac{D k_{E}}{\operatorname{Re} S c_{E}}\right] \rho^{*} S_{Q}^{*} \\
& \frac{\partial}{\partial x_{i}^{*}}\left(\varepsilon \frac{\partial V^{*}}{\partial x_{i}^{*}}\right)=-Q^{*}
\end{aligned}
$$

$$
\begin{aligned}
& E_{i}^{*}=-\frac{\partial V^{*}}{\partial x_{i}^{*}} \\
& \frac{\partial U_{i}^{*}}{\partial x_{i}^{*}}=0 \\
& \frac{\partial}{\partial t^{*}}\left(U_{i}^{*}\right)+\frac{\partial}{\partial x_{j}}\left(U_{i}^{*} U_{j}^{*}\right)=-\frac{\partial p^{*}}{\partial x_{i}{ }^{*}}+\frac{1}{\operatorname{Re}}\left(\frac{\partial^{2} U_{i}^{*}}{\partial x_{j}^{*} \partial x_{j}^{*}}\right) \\
& +\frac{G r_{E}}{\operatorname{Re}^{2}} Q^{*} E_{i}^{*}
\end{aligned}
$$

where for the free flow case where $\mathrm{U}_{0}=\kappa \mathrm{E}_{0}$, the charge transport and momentum equations may be re-written as:

$$
\begin{aligned}
& \frac{\partial}{\partial t^{*}}\left(Q^{*}\right)+\frac{\partial}{\partial x_{i}{ }^{*}}\left(Q^{*} U_{i}^{*}\right)=\frac{1}{\operatorname{Re}_{E} S c_{E}} \frac{\partial}{\partial x_{i}}\left(D^{*} \frac{\partial Q^{*}}{\partial x_{i}{ }^{*}}\right)- \\
& \left(E_{i}^{*} \frac{\partial Q^{*}}{\partial x_{i}^{*}}+Q^{* 2}\right)+\left[\frac{D k_{E}}{\operatorname{Re}_{E} S c_{E}}\right] \rho^{*} S_{Q}^{*}
\end{aligned}
$$

$$
\begin{aligned}
& \frac{\partial}{\partial t^{*}}\left(U_{i}^{*}\right)+\frac{\partial}{\partial x_{j}}\left(U_{i}^{*} U_{j}^{*}\right)=-\frac{\partial p^{*}}{\partial x_{i}^{*}}+\frac{1}{\operatorname{Re}_{E}}\left(\frac{\partial^{2} U_{i}^{*}}{\partial x_{j}^{*} \partial x_{j}^{*}}\right) \\
& +C M^{2} Q^{*} E_{i}^{*}
\end{aligned}
$$

In all of the non-dimensional equations the asterisk denotes a non-dimensional value while a zero subscript indicates a reference value, and in the non-dimensional equations, $\rho^{*}$ is equal to unity. Equation (1) is the conservation of space charge equation for a pure unipolar species, where $\mathrm{Q}$ is space charge, $\mathrm{t}$ is time, $\mathrm{U}$ is velocity, $\mathrm{x}$ is position, $\mathrm{\kappa}$ is ionic mobility, $\mathrm{E}$ is electric field and $\mathrm{S}$ is the charge injection source term (the injected current). The reader should consult the references [24-26] for the definition of the electrical Schmidt number $\mathrm{Sc}_{\mathrm{E}}$. The electrical Damkohler number, $\mathrm{Dk}_{\mathrm{E}}$ is defined 
by the ratio of the reaction speed to the diffusion speed, and is given by equation (8), where $\mathrm{D}$ is the diffusion coefficient. In equation $1, \operatorname{Re}$ is a conventional Reynolds number.

$$
\left[\frac{S_{Q o} x_{o}^{2}}{D_{o} Q_{o}}\right]
$$

Equation (2) is the Poisson equation for the voltage (V) where it has been assumed that $\mathrm{V}_{0} / \mathrm{x}_{0}{ }^{2}$ scales with $\mathrm{Q}_{0} / \varepsilon_{0}$, and equation (3) is the definition of the electric field (E). The hydrodynamic equations are the conservation of mass and the EHD conservation of momentum given by equations (4) and (5) respectively, where $\mu$ is dynamic viscosity and $\rho$ is density. Equation (5) is the conventional conservation of momentum equation with an added source term which accounts for the Lorentz force due to the presence of space charge and electric field. The non-dimensional number, $\mathrm{Gr}_{\mathrm{E}}$ is the electrical Grashof number defined and discussed in [26]. Equations (6) and (7) are the charge transport and momentum equations for the free flow case respectively, where all the non-dimensional numbers are fully discussed in [26]. Here, we neglect dielectrophoretic and electrostrictive forces as they are negligible for the simulation parameters that are used in this study [24-26].

Equation (7) is the common form of the non-dimensional momentum conservation equation, but only strictly relevant to free rather than forced convection systems, by virtue of the $\mathrm{U}_{0}=\kappa \mathrm{E}_{0}$ scaling. In the non-homogeneous EHD forced flow system here, since both hydrodynamic $\left(\mathrm{U}_{0}\right)$ and electrical $\left(\kappa E_{0}\right)$ velocity scales exist, we follow the traditional engineering approach as given in equations (1)-(5).

\subsection{CODE DETAILS}

The problem was simulated using a two dimensional axisymmetric, non-uniform, finite volume mesh. Time was discretised using a second order Crank-Nicholson scheme and discretisation in space was achieved using a hybrid scheme $[27,28]$. Unfortunately, in order to assure variables are conserved, the implicit component of the flux is mainly calculated using the first order upwind scheme $[27,28]$ in non-smooth regions. This implies a first order accuracy in regions such as those close to the injection location. Such a scheme is numerically diffusive and thus produces results that may seem unrealistic, which is one disadvantage of the code used here.

A back staggered grid arrangement was used for the momentum and scalar control volumes and the PISO scheme $[27,28]$ is used to solve the coupled pressure and momentum fields. For similar reasons as with pressure and velocity coupling, the charge and electric field values are stored at different locations. The non-linear ionic drift term was implicitly discretized in a manner explained fully elsewhere [27,28]. A second order interpolation scheme is employed to calculate the electric field at the face. Full details of the code may be found elsewhere [27, 28].

\section{MESH DETAILS AND INPUT PARAMETERS}

In this section, the mesh and boundary conditions used shall be presented, followed by the physical properties used. These properties shall be used in order to calculate relevant nondimensional numbers that will help place the problem in a specific regime, and will also assist in examining the degree of non-homogeneity present in the fluid.

\subsection{THE MESH AND BOUNDARY CONDITIONS}

Figure 2 shows the non-uniform mesh used in the study with the arrows indicating the direction of flow. Cells are more refined closer to the walls and a particularly fine region exists where the largest velocities are expected, close to the orifice, and in between the electrode and orifice plate. At the region above the electrode, 50x50 nodes were used, between the electrode and grounded orifice 38 nodes were employed (along the $\mathrm{x}$ direction) and at the orifice: 50 (y-direction) x 150 (x-direction) nodes were used.

The injection of charge was simulated by fixing a single control volume at the corner of the electrode to a constant level of charge per unit time, as shown in Figure 2. The value chosen was based on experimental data taken from Diesel fuel pumped through an atomizer of the same geometry. Figure 3 shows some sample experimental data taken at $\mathrm{L} / \mathrm{d}$ ratios $=0.6-2$ [29]. There is an initial linear region and a non-linear region, the former operating under an Ohmic regime and the latter under a chaotic charge injection regime [2-16].

The current values used in our study correspond to three different points for an $\mathrm{L} / \mathrm{d}=0.5$ interpolated from the data of Figure 3. The three data points used in this paper are representative of an Ohmic regime, a 'transitional' regime and one of a 'chaotic' regime. Specifically, we used voltage values of $1.7 \mathrm{kV}, 3 \mathrm{kV}$ and $4.5 \mathrm{kV}$ corresponding to currents of $.66 \mu \mathrm{A}, 2.63 \mu \mathrm{A}$ and $8.62 \mu \mathrm{A}$ respectively [29]. The ' $\mathrm{L}$ ' for the atomizer used is equal to $125 \mu \mathrm{m}$ and the diameter $\mathrm{d}$, is $250 \mu \mathrm{m}$. These values shall be utilized in section 3.2 for calculation of various quantities of interest.

Other boundary conditions include no-slip at the walls, an inlet velocity equal to $0.06944 \mathrm{~m} / \mathrm{s}$, giving a mean injection velocity at the orifice equal to $10 \mathrm{~m} / \mathrm{s}$. The voltage was fixed at the electrode, at a value which depended on the regime being simulated and a voltage $=0$ was imposed at the grounded orifice plate surface. Charge density was fixed to zero at the electrode wall, and a charge flux equal to zero was employed on the grounded surface and atomizer outlet.

\subsection{PHYSICAL PROPERTIES AND THEORETICAL NON-DIMENSIONAL NUMBERS}

As stated previously, the fuel used in this study was Diesel whose main component is dodecane. Relative permittivity for this fuel is equal to 2.2 , dynamic viscosity to $.001 \mathrm{~kg} / \mathrm{ms}$, density to $800 \mathrm{~kg} / \mathrm{m}^{3}$ and ionic mobility is equal to $1 \times 10^{-7}$ $\mathrm{m}^{2} / \mathrm{Vs}[2]$. 


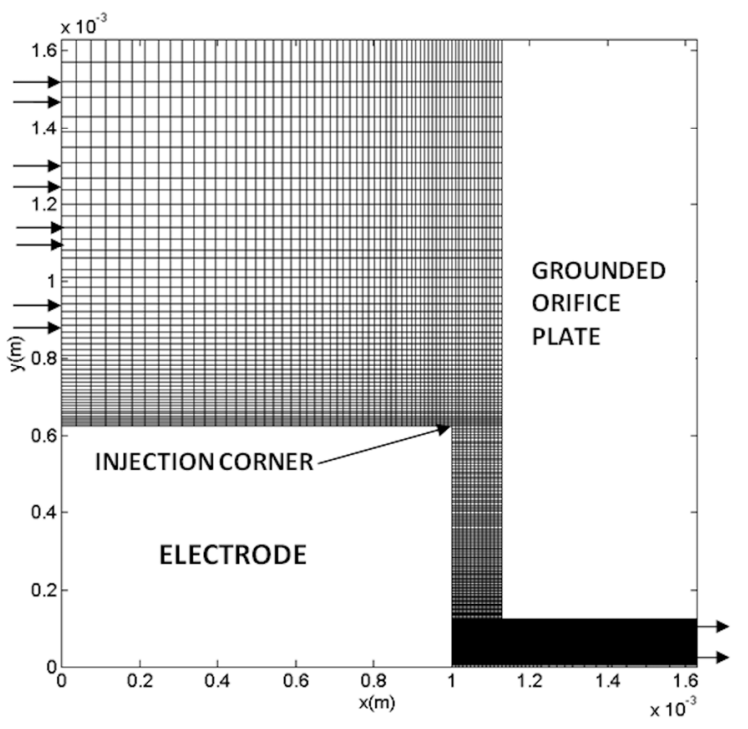

Figure 2. Non-uniform mesh applied to 'Region of interest' from Figure 1, showing the flow direction as being from left to right. The high voltage electrode is a region of fixed voltage as is the grounded orifice plate.

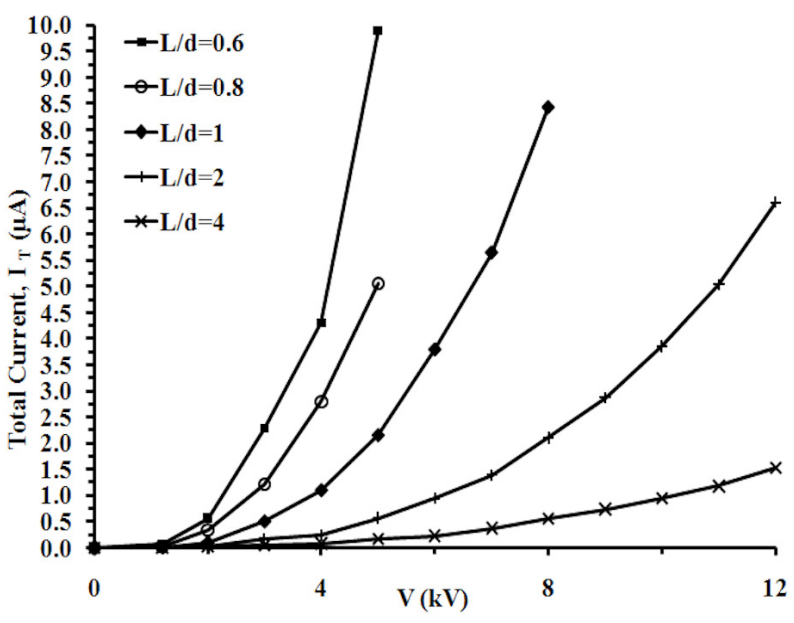

Figure 3. $\mathrm{I}_{\mathrm{T}} \mathrm{vs} \mathrm{V}$ characteristics for a steady flow charge injection atomizer using a tungsten sharp edge electrode, Diesel fuel, an orifice diameter $=250 \mu \mathrm{m}$ with $<\mathrm{u}_{\text {inj }}>=10 \mathrm{~m} / \mathrm{s}$. [29]

Using the properties of Diesel and the geometry of the charge injection atomizer, we can calculate a number of nondimensional numbers which will help place the simulations within nominal regimes. Table 1 shall provide seven such parameters: the $\mathrm{T}, \mathrm{C}, \mathrm{M}, 1 / \mathrm{Re}_{\mathrm{E}}$, and $\mathrm{CM}^{2}$ numbers which appeared in equation (7) and the $\mathrm{Dk}_{\mathrm{E}} / \mathrm{Re}_{\mathrm{E}} \mathrm{Sc}_{\mathrm{E}}$ and $1 / \mathrm{Re}_{\mathrm{E}} \mathrm{Sc}_{\mathrm{E}}$ parameters which appeared in equation (6).

The T,C, and $\mathrm{M}$ parameters may be calculated using equation set (8).

The $\mathrm{T}$ parameter is defined by Castellanos as an equivalent electrical Rayleigh number [24] and is a measure of instability in the system. The $\mathrm{C}$ parameter, or charge injection parameter, is defined by Castellanos [24] as a measure of the strength of charge injection, where $\mathrm{C}>>1$ indicates strong injection. The M parameter is described by Atten [21] as a measure of EHD turbulence within the system and may also be related to the electrical Reynolds number which appeared in equation (7) [21].

$$
T=\frac{\varepsilon_{0} V_{0}}{\kappa_{0} \mu_{0}}, C=\frac{Q_{0} x_{0}^{2}}{\varepsilon_{0} V_{0}}, M=\left(\frac{T}{\operatorname{Re}_{E}}\right)^{1 / 2}=\frac{\left(\varepsilon_{0} / \rho_{0}\right)^{1 / 2}}{\kappa}
$$

These three non-dimensional values have been used by researchers [20-24] to quantify the instability of a quiescent system, and they appeared in the free flow momentum equation (equation (7)). However, the T, C, M analysis is technically invalid here which makes it necessary to discuss non-dimensional numbers appearing in the forced flow case. Numbers appearing in the transport equations (equations (1) and (5)) are now $1 / \operatorname{Re}_{\mathrm{E}} \mathrm{Sc}_{\mathrm{E}}, \kappa_{0} \mathrm{E}_{0} / \mathrm{U}_{0}, \mathrm{Dk}_{\mathrm{E}} / \operatorname{ReSc}_{\mathrm{E}}, 1 / \mathrm{Re}$, and $\mathrm{Gr}_{\mathrm{E}} / \mathrm{Re}^{2}$ and these are provided in table 2 .

Table 1. Non-dimensional parameters according to regime. (free-flow at corner).

\begin{tabular}{|l|l|l|l|l|l|l|l|}
\hline $\mathbf{V ~}(\mathbf{k V})$ & $\mathbf{T}$ & $\mathbf{C}$ & $\mathbf{M}$ & $\mathbf{C} \mathbf{M}^{\mathbf{2}}$ & $\mathbf{1} / \mathbf{R e}_{\mathrm{E}}$ & $\frac{\mathbf{D k}_{\mathrm{E}}}{\mathbf{R e}_{\mathrm{E}} \mathbf{S c}_{\mathbf{E}}}$ & $\begin{array}{l}\mathbf{1} \\
\mathbf{R e}_{\mathrm{E}} \mathbf{S c}_{\mathrm{E}}\end{array}$ \\
\hline 1.7 & 320 & 0.7 & 1.5 & 1.6 & 0.007 & $4.5 \mathrm{e}-11$ & $1.5 \mathrm{e}-5$ \\
\hline 3.0 & 564 & 1.5 & 1.5 & 3.4 & 0.004 & $2.6 \mathrm{e}-11$ & $8.3 \mathrm{e}-6$ \\
\hline 4.5 & 846 & 3.2 & 1.5 & 7.2 & 0.003 & $1.7 \mathrm{e}-11$ & $5.6 \mathrm{e}-6$ \\
\hline
\end{tabular}

Table 2. Non-dimensional parameters according to regime. (forced-flow at corner).

\begin{tabular}{|c|c|c|c|c|c|}
\hline \multirow[t]{2}{*}{$\mathrm{V}(\mathrm{kV})$} & 1 & $\mathbf{D k}_{\mathrm{E}}$ & 1 & $\mathbf{G r}_{\mathbf{E}}$ & \multirow{2}{*}{$\begin{array}{c}\boldsymbol{\kappa}_{0} \mathbf{E}_{0} \\
\mathbf{U}_{0}\end{array}$} \\
\hline & $\operatorname{ReSc}_{\mathrm{E}}$ & $\operatorname{ReSc}_{\mathrm{E}}$ & $\operatorname{Re}$ & $\mathbf{R e}^{2}$ & \\
\hline 1.7 & $2.7 e-5$ & $3.1 \mathrm{e}-6$ & 0.01 & 5.1 & 1.8 \\
\hline 3.0 & $2.7 e-5$ & $3.1 \mathrm{e}-6$ & 0.01 & 35.7 & 3.2 \\
\hline 4.5 & $2.7 e-5$ & $3.1 \mathrm{e}-6$ & 0.01 & 176.0 & 4.8 \\
\hline
\end{tabular}

In Table $1, \mathrm{Q}$ has been calculated using a value of $Q=I_{T} / \dot{\tau}$ where $\dot{\tau}$ is the volume flow-rate, and $\mathrm{I}_{\mathrm{T}}$ is the current from Figure 3 where for the Ohmic case, transitional case and chaotic case, $\mathrm{Q}_{0}$ is $1.34 \mathrm{C} / \mathrm{m}^{3}, 5.36 \mathrm{C} / \mathrm{m}^{3}$ and 17.6 $\mathrm{C} / \mathrm{m}^{3}$ respectively. The value of $\mathrm{U}_{0}$ was calculated as the average velocity between the electrode corner and grounded orifice plate, equal to $0.75 \mathrm{~m} / \mathrm{s}$. This region was chosen as it is the location of charge injection, and is thus of greatest interest for the initial discussion carried out in this section.

The theoretical results of table 1 have assisted in placing the atomizer in particular regimes. Quite clearly, the atomizer is operating in an unstable regime for the transitional and chaotic cases, as $\mathrm{C}>>1$ and $\mathrm{T}>160$ [24], however for the experimental Ohmic case the system is on the borderline of theoretical instability. In the free flow momentum case it can be seen that the Lorentz force term is the most significant contributor to momentum transport for all of the regimes, as can be seen by the value of $\mathrm{CM}^{2}$, while diffusion is negligible. Charge transport is mainly attributed to ionic drift and convective transport as the charge injection source $\left(\mathrm{Dk}_{\mathrm{E}} / \mathrm{ReSc}_{\mathrm{E}}\right)$ and charge diffusion $\left(1 / \mathrm{Re}_{\mathrm{E}} \mathrm{Sc}_{\mathrm{E}}\right)$ terms are negligible.

In Table 2, as with the free flow case the Lorentz force contribution from equation (5) $\left(\mathrm{Gr}_{\mathrm{E}} / \mathrm{Re}^{2}\right)$ is the strongest contributor to momentum transport and becomes even more 
relevant at higher applied voltages. Diffusion $(1 / \mathrm{Re})$ seems to have a larger impact in the forced flow case than in the free flow case. Observing the non-dimensional terms from equation (1), we see the ionic drift term $\left(\kappa_{0} \mathrm{E}_{0} / \mathrm{U}_{0}\right)$ as a major contributor to charge transport, this time with values greater than 1 as opposed to the free flow case. Furthermore, as with the free flow case, the source term and diffusion term magnitudes, defined by $\mathrm{Dk}_{\mathrm{E}} / \mathrm{ReSc}_{\mathrm{E}}$ and $1 / \mathrm{ReSc}_{\mathrm{E}}$ respectively, are negligible.

A final theoretical calculation of particular relevance to our problem is to calculate the non-dimensional numbers as a function of position within the atomizer, for the forced flow case. Table 3 presents the non-dimensional numbers from Table 2, for the chaotic regimes at the electrode gap $(y=0.4$ $\mathrm{mm}, \mathrm{x}=1.05 \mathrm{~mm})$ and orifice channel $(\mathrm{x}=0.0013 \mathrm{~m}$, $\mathrm{y}=0.0005 \mathrm{~m}$ ), see Figure 2 for details on the positions. $\mathrm{Q}_{0}$ at the mid electrode position has been calculated from the simulations, while $\mathrm{Q}_{0}$ at the orifice is based on experimental data of spray specific charge [29] $\left(0.27 \mathrm{C} / \mathrm{m}^{3}\right)$, as this is more accurate than the dissipative simulation calculation at that location. The velocities have been extracted from the simulation data as $5 \mathrm{~m} / \mathrm{s}$ at the orifice position and $0.5 \mathrm{~m} / \mathrm{s}$ at the electrode gap.

Table 3. Non-dimensional parameters according to position. (forced-flow and chaotic),

\begin{tabular}{|c|c|c|c|c|c|}
\hline \multirow[t]{2}{*}{ Position } & 1 & $\mathbf{D k}_{\mathbf{E}}$ & 1 & $\mathbf{G r}_{\frac{2}{2}}$ & $\kappa_{0} \mathbf{E}_{0}$ \\
\hline & $\operatorname{ReSc}_{\mathrm{E}}$ & $\operatorname{ReSc}_{\mathrm{E}}$ & $\operatorname{Re}$ & & \\
\hline Electrode Gap & $5 e-5$ & $7.5 \mathrm{e}-10$ & 0.02 & 9.0 & 7.2 \\
\hline Orifice channel & $4 \mathrm{e}-6$ & $8.3 e-10$ & 0.002 & 0.06 & 0.52 \\
\hline
\end{tabular}

The simple theoretical analysis here has quantified the effect of various terms appearing in the transport equations for both the free flow and forced flow cases at different regimes and positions. The non-homogeneity of the problem is made apparent, showing that near the orifice electrical forces contribute less than hydrodynamic forces, and we expect to acquire similar results from the simulation results. The theoretical results presented here also suggest that we should be able to observe a notable increase in instability while going from one regime to the next, while also when moving from one position to the next.

We reach these conclusions mainly by observation of the Lorentz force contribution to the momentum, which is very significant for all cases, but also by observation of the T, C and $\mathrm{M}$ parameters, which indicate that the atomizer operates in an unstable regime.

\section{RESULTS}

The simulation results to be displayed shall demonstrate how the internal flow of the atomizer changes according to charging level and position and this shall be done by calculation of the non-dimensional terms that we discussed in section 3. Firstly, the base flow pattern with no charge injection shall be presented followed by the flow pattern for the three regimes discussed.

\subsection{BASE FLOW PATTERN}

Figure 4 shows the base flow pattern within the atomizer, indicating that the effect of a sharp change in velocity due to the presence of the electrode sharp corner does not cause any notable production of turbulence within the internal geometry.

We now investigate the effect of space charge on the flow of the dielectric.

\subsection{OHMIC REGIME}

As stated in the introduction, the formation of ions in the dielectric is attributed to what is most likely an electrochemical mechanism that is still not well understood. In the charge conservation equation, there are three terms which contribute to the movement of these ions, and these are a bulk convective flux term, a diffusive flux term and an ionic drift term.

The bulk convective flux term $\rho Q U_{i}$ is dominant where bulk flow is greatest, e.g. at the exit of the atomizer, where velocities are high, while ionic drift $\rho \kappa \mathrm{QE}_{\mathrm{i}}$ is dominant where bulk flow is minimal, and the scaling analysis we shall carry out further on shall show exactly this.

As stated in section 3.2, the work here shall discuss spatial non-homogeneity in the fluid or the relevant contribution of electrical and hydrodynamic terms at different locations in the atomizer. In the charge injection device, bulk flow is minimal before the fluid enters the region under the electrode and at that location, we would expect a recirculation zone which would not only be attributed to a drastic change in pressure, but would also be a result of electroconvection. From the results to be displayed in this section, the reader will observe how the relative magnitudes of $\rho \kappa \mathrm{QE}_{\mathrm{i}}$ and $\rho \mathrm{QU} \mathrm{U}_{\mathrm{i}}$ affect the momentum field of the dielectric liquid.

Theoretically, the linear regime, which we have termed an Ohmic regime, should not produce any notable instabilities as the level of chaotic charge injection is negligible, thus resulting to no roll structures, as the charge injected is not enough to initiate instability. This was also observed in table 1 for the Ohmic $(1.7 \mathrm{kV})$ case.

In Figure 5, at the injecting electrode, a small disturbance is observed, attributed to the Lorentz force in the conservation of momentum equation, while also due to the fact that the value of $\kappa E_{i}$ is comparable to the mean bulk flow, $U_{i}$ close to the electrode. For the Ohmic regime, at the region close to the electrode corner, $\left\langle\left|U_{i}\right|>|<| \wedge E_{i}\right|>\sim 2$.

On the contrary, where the convective contribution due to bulk flow is much larger than the ionic drift contribution, as in the orifice channel, where the value of $\left\langle\left|U_{i}\right|>\right|\left\langle\left|\kappa E_{i}\right|>\sim 10\right.$, then little to no instabilities are observed, as is the case seen in Figure 5 .

\subsection{TRANSITIONAL AND CHAOTIC REGIMES}

Figures 6 and 7 show the flow pattern within the atomizer for the case of a transitional regime and fully chaotic regime respectively. The transitional and chaotic regimes are observed experimentally via a sharp increase in the total 
injected current. By observation of the simulation results in this section, it can be seen that this non-linear increase in charge level greatly affects the operation of the atomizer and produces a clear recirculation zone. In quiescent flow problems discussed elsewhere [20-24], the mechanism by which these roll type instabilities appear is fully discussed. In both Figures 6 and 7, the effect of space charge on the forced flow pattern is clearly seen, and its non-homogeneity is also very apparent. By inspection of Figure 6, we see a chaotic region forming locally around the injection point, which from a practical point of view means that less charge carriers will get flushed out of the atomizer.

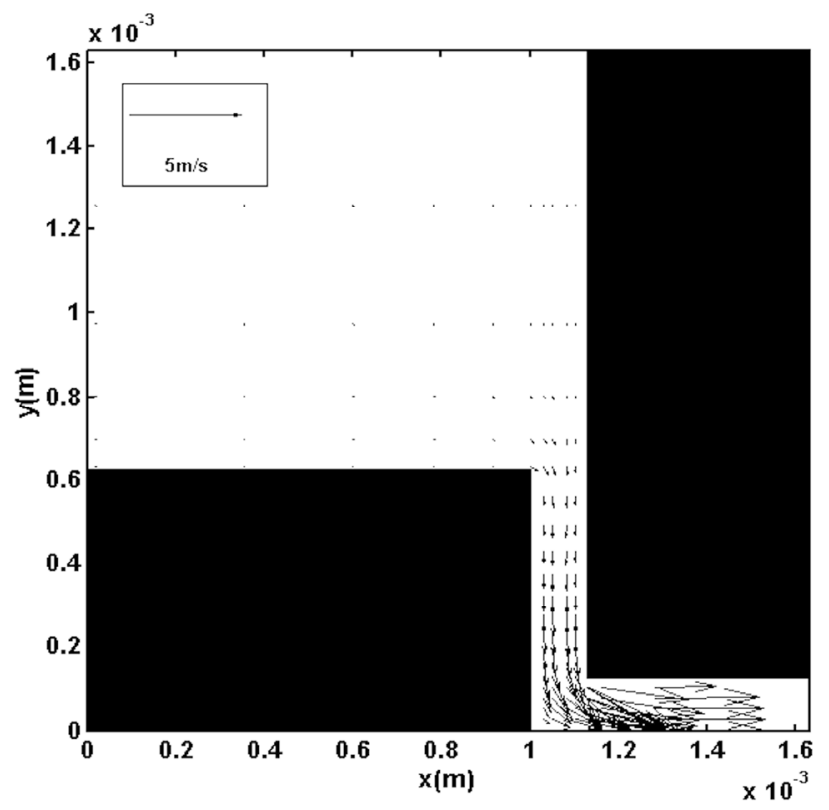

Figure 4. Base flow pattern with no charge injection

At the region of the electrode corner, for the transitional regime shown in Figure 6, $<\left|U_{i}\right|>|<|\left\langle E_{i}\right|>\sim 3$, showing again, as in the linear regime, that the coupling between the bulk flow and ionic drift is very strong. At other points in the geometry, the ionic drift contribution is larger, for example at a y-position $=0.4 \times 10^{-3} \mathrm{~m}$, the value of $<\left|U_{i}\right|>/<\left|\kappa E_{i}\right|>\sim 0.7$.

EHD turbulence production becomes even more acute for higher values of space charge, where in Figure 7 , the fluid has begun to flow away from the inter-electrode gap entirely. This is problematic, as it suggests that as charging level is increased, a larger injection pressure is needed to flush away the charge carriers from inside the atomizer. We postulate that this level of turbulence within the atomizer may have an effect on the quality of the spray produced.

As with the transitional regime, by comparing the relative magnitude of $U_{i}$ and $\kappa E_{i}$ at the corner, we find a strong coupling between hydrodynamic and electrical drift where,

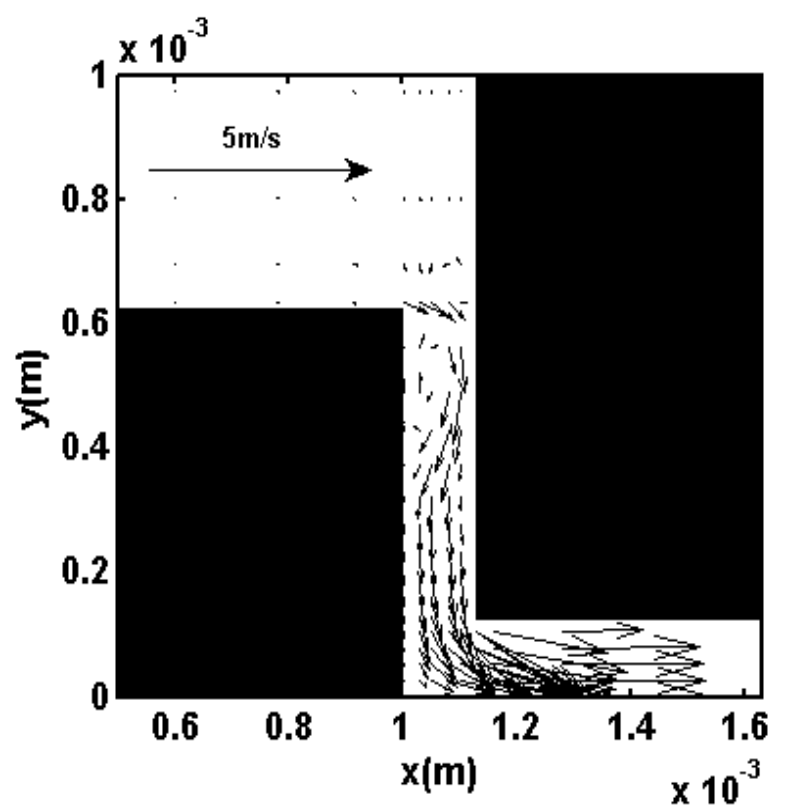

Figure 5. Flow pattern for linear regime. $\mathrm{V}=1.7 \mathrm{kV}$ and $\mathrm{I}=0.66 \mu \mathrm{C} / \mathrm{s}$

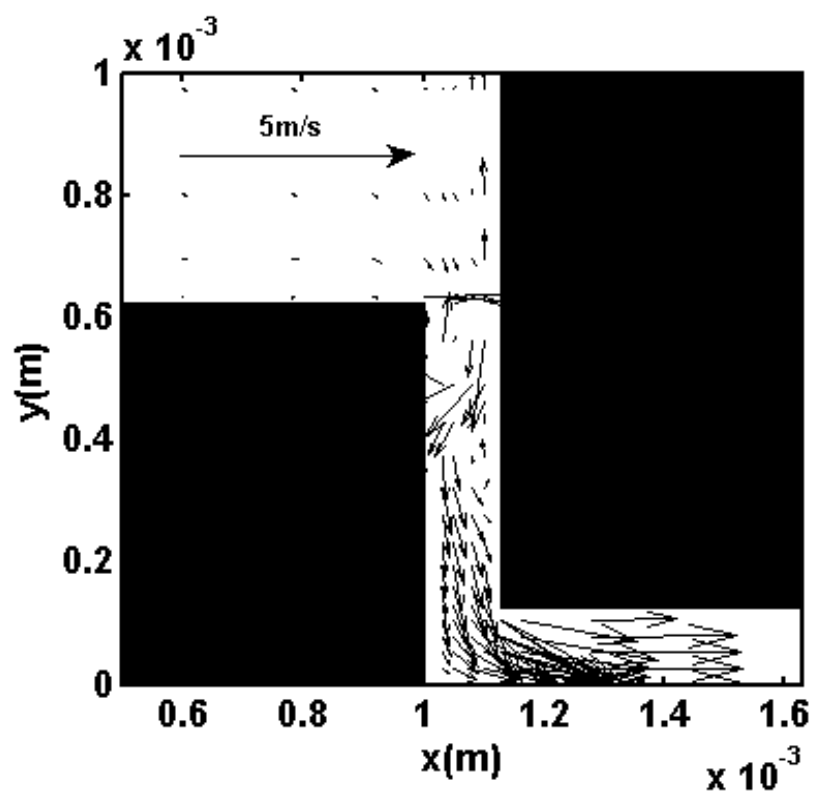

Figure 6. Flow pattern for transitional regime. $\mathrm{V}=3.0 \mathrm{kV}$ and $\mathrm{I}=2.63 \mu \mathrm{C} / \mathrm{s}$

$<\left|U_{i}\right|>|<|\left\langle E_{i}\right|>\sim 2$ while at the fixed $\mathrm{y}=0.4 \mathrm{~mm}$ we find a larger ionic drift contribution where $<\left|U_{i}\right|>/<\left|k E_{i}\right|>0.5$. A further investigation that will clarify where hydrodynamic and electrical forces dominate is by observing the time averaged electrical $\left(1 / 2 \varepsilon<E_{i}>^{2}\right)$ and kinetic $\left(1 / 2 \rho<U_{i}>^{2}\right)$ energies at different locations in the domain, and we do this here for the chaotic case. The reader should note that averaging is performed at a single control volume under statistically stationary conditions.

At the electrode corner, $\varepsilon<E_{i}>^{2} / \rho<U_{i}>^{2} \sim 0.6$ showing that these energies are of the same order of magnitude, 
indicating that both the electrical and kinetic energies contribute at that location. Moving to a position at the middle of the electrode gap $(y=0.4 \mathrm{~mm}, x=1.05 \mathrm{~mm})$ the electrical energy contribution is larger, with $\varepsilon<E_{i}>^{2} / \rho<U_{i}>^{2} \sim 8.4$ indicating that electrical forces in the electrode gap are hindering the acceleration of the fluid out of the atomizer, decreasing its kinetic energy. Furthermore, moving to the orifice channel, $(x=0.0013 \mathrm{~m}, \mathrm{y}=0.0005 \mathrm{~m})$ the relative contribution is drastically different, with $\varepsilon<E_{i}>^{2} / \rho<U_{i}>^{2} \sim 0.003$, indicating a location of almost pure hydrodynamic contribution. Based on these results, the very strong flow non-homogeneity present in the charge injection atomizer is made apparent and the invalidity of conventional 1-way coupled simulations for this type of fluid and geometry is confirmed.

Having observed the flow pattern within such devices it is necessary to comment on whether or not these results may be considered as realistic. The one major drawback is that we have assumed a single charge injection point at the corner of the electrode, and while this is a reasonable assumption for initial two dimensional simulations, the fact that the charge will remain constant is not particularly realistic. A charge injection model of the form $\mathrm{I}=\mathrm{A}\left(\mathrm{E}-\mathrm{E}_{\mathrm{TH}}\right)^{\mathrm{n}}$ must be defined which will locally adapt the flux injected on each electrode surface. This is because the space charge field that develops within the liquid will serve to alter the electric field imposed on the liquid, which must be taken into consideration in more complex simulations.

Furthermore, it was mentioned in section 2.1 that the spatial discretisation scheme used in non-smooth regions was only of first order in order to maintain a conserved solution. This leads to numerical diffusion [30], which in the physical sense is manifested as dissipation of the space charge, downstream of the electrode. While this does generate high errors near the orifice, it is still accurate close to the electrode, which is the region of greatest interest as far as EHD chaos is concerned.

\subsection{KOLMOGOROV SCALES}

Having discussed the main results from the simulations it is necessary to state whether or not the analysis is in fact a direct numerical simulation (DNS). The main source of energy is the electrical energy $=1 / 2 \varepsilon \mathrm{E}_{0}^{2},[24]$ where $\varepsilon$ is the electrical permittivity. The relevant kolmogorov length $(\eta)$ and timescale $(\tau)$ may then be calculated by equating the input electrical energy to the rate of dissipation $\varepsilon_{\mathrm{dp}}$ of the turbulent kinetic energy, and then by utilizing equations (9) and (10). The input kinetic energy has been neglected for these calculations, as from observation of Figure 4 it can be seen that there are no fluctuations which are attributed to input hydrodynamic energy. This suggests that there is negligible turbulence production due to hydrodynamic forces alone.

For the chaotic case, the Kolmogorov length $\eta=3.53 \times 10^{-}$ ${ }^{6} \mathrm{~m}$ and the time $\tau=1 \times 10^{-5} \mathrm{~s}$. Between the electrode and grounded orifice plate, the mean cell size of the mesh was 9 x $10^{-6} \mathrm{~m}$ and the time step employed $=1 \times 10^{-5} \mathrm{~s}$. DNS has been carried out by a number of researchers at cell sizes $\sim 2 \eta$ and larger, such as in thermal convection [31] and fundamental DNS studies [32-34] for 3D simulations, suggesting that the simulation here does adequately capture the smallest length and time scales.

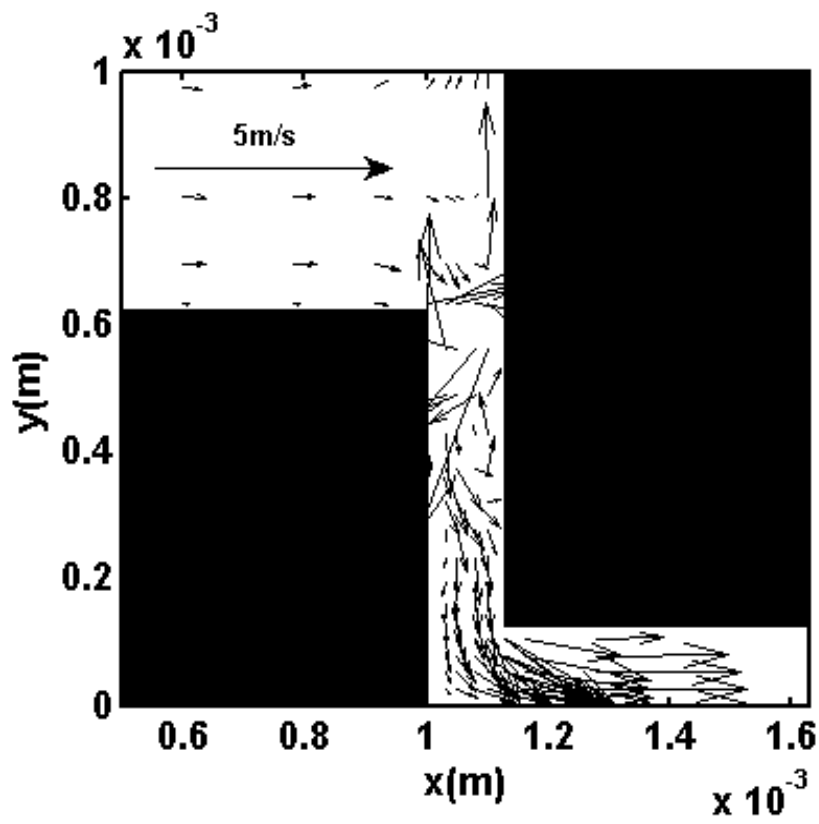

Figure 7. Flow pattern for chaotic regime. $\mathrm{V}=4.5 \mathrm{kV}$ and $\mathrm{I}=8.62 \mu \mathrm{C} / \mathrm{s}$

$$
\begin{aligned}
& \eta=\left(\frac{v^{3}}{\varepsilon_{d p}}\right)^{1 / 4} \\
& \tau=\left(\frac{v}{\varepsilon_{d p}}\right)^{1 / 2}
\end{aligned}
$$

Furthermore, the mesh is adequate for the purposes of our study as the simulation has correctly predicted the transition to chaos based on both the experimental data and the theoretical non-dimensional numbers calculated.

Finally, this is a two dimensional simulation which does not allow for the full production of turbulence that would take place under more realistic three-dimensional conditions. This means that the simulation presented here is not strictly one that approximates the fully turbulent nature of EHD flow very accurately, giving further reason to employ cell sizes slightly larger than the Kolmogorov length. What was of real interest in this study was to employ a 2-way coupled code in order to investigate the transition to chaos and flow non-homogeneity in the internal geometry of a charge injection atomizer, and this was done successfully.

\section{CONCLUSIONS}

The internal flow pattern of a typical charge injection atomizer geometry has been simulated in two dimensions 
using a coupled space charge and momentum field for the first time.

When the atomizer operates in the linear or Ohmic regime, the flow is only slightly unstable, with a disturbance appearing locally around the electrode. However, at higher values of injected space charge corresponding to the experimental nonlinear regime, results have revealed that EHD chaos in the liquid is significant.

Given the data presented, we have reason to believe that the turbulence generated will affect the performance of the atomizer as it tends to cause a disturbance in the flow field which may affect the flushing out of charge carriers. This would reduce the level of charge carried out to the atomization zone.

In order to better quantify this generated instability, a more accurate model describing the injection of charge must be developed and used in order to implement more accurate boundary conditions. Such a model would be based on the wide range of experimental data available in the literature [216,28]. Finally, for a more accurate analysis of the space charge distribution far downstream of the charge injection location, a less dissipative scheme should be employed.

\section{REFERENCES}

[1] A.G. Bailey, "The Theory and Practice of Electrostatic Spraying", Atomization Spray Technology, vol. 2, pp. 95-134, 1986.

[2] J.S. Shrimpton, Electrostatic Atomization and Combustion of Hydrocarbon Oils, Ph.D. thesis, UMIST, Manchester, UK, 1995

[3] A.R.H Rigit, Performance of a Charge Injection Electrostatic Atomizer and Spray Characteristics, Ph.D. thesis, Imperial College London, London, UK, 2003.

[4] A.R.H Rigit and J.S. Shrimpton, "Electrical Performance of Charge Injection Electrostatic Atomizers", Atomization and Sprays, Vol. 16, pp. 401-419, 2006.

[5] A.R.H Rigit and J.S. Shrimpton, "Spray Characteristics of Charge Injection Electrostatic Atomizers with Small-Orifice Diameters", Atomization and Sprays, Vol. 16, pp. 421-442, 2006.

[6] J.S. Shrimpton and A.J. Yule, "Characterization of charged hydrocarbon sprays for application in combustion systems", Experiments in Fluids Vol. 26, pp 460-469, 1999.

[7] G AL-Ahmad, J.S. Shrimpton, E.L. Ergene and F. Mashayek, "Electrical Performance of a Charge Injection Atomizer using Viscous Organic Oils", Atomization and Sprays, Vol. 19, pp.547$566,2009$.

[8] K. Kim and J. Turnbull, "Generation of charged drops of insulating liquids by electrostatic spraying”, J. Appl. Phys., Vol. 47, pp. 19641969, 1976.

[9] K.S. Robinson, R.J. Turnbull, and K. Kim, "Electrostatic spraying of liquid insulators", Trans. IEEE Ind. App. Sys. Vol. 16, pp. 308-317, 1980.

[10] A.J. Kelly, "The electrostatic atomization of hydrocarbons", J. Institute of Energy, pp. 312-320, 1984.

[11] A.J. Kelly, "Electrostatic Atomizing Device", US Patent 4,255,777, March, 1981.

[12] A.J. Kelly, "Electrostatic Atomizing Device", US Patent 4,380,786, April, 1983.

[13] A.J. Yule, J.S. Shrimpton, A.P. Watkins, W.Balachandran, and D.Hu, "Electrostatically atomized hydrocarbon sprays", Fuel, Vol. 74, pp. 1094-1103, 1995.

[14] J.S. Shrimpton and A.J. Yule, "Design issues concerning charge injection atomizers", Atomization and Sprays, Vol. 14, pp. 127-142, 2004.

[15] H. Romat and A. Badri, "Internal electrification of Diesel oil injectors", J. Electrostatics, Vol. 51-52, pp. 481-487. 2001

[16] A. Kourmatzis, J. Allen, and J.S. Shrimpton, "Electrical and spray characteristics of a multi-orifice charge injection atomizer for electrically insulating liquids", Atomization and Sprays, Vol. 20, pp. 269-280, 2010.

[17] A. Alj, A. Denat, J. P. Gosse, and B. Gosse, "Creation of charge carriers in nonpolar liquids", IEEE Trans. Electr. Insul., Vol. 20, pp. 221-231, 1985

[18] A.R.H. Rigit and J.S. Shrimpton, "Estimation of the diameter-charge distribution in polydisperse electrically charged sprays of electrically insulating liquids", Experiments in Fluids, Vol. 46, pp. 1159-1171, 2009.

[19] J.S. Shrimpton and A.J. Yule, "Electrohydrodynamics of charge injection atomization: Regimes and fundamental limits", Atomization and Sprays, Vol. 13, pp. 173-190, 2003.

[20] A. Castellanos, "Coulomb-Driven convection in electrohydrodynamics", IEEE Trans. Electr. Insul., Vol. 26, pp. 1201-1215, 1991.

[21] P. Atten., "Electrohydrodynamic instability and motion induced by injected space charge in insulating liquids", IEEE Trans. Dielectr. Electr. Insul., Vol. 3, pp. 1-17, 1996.

[22] P. A. Vazquez, G. E. Gheorgiou, A. Castellanos., "Numerical analysis of the stability of the electrohydrodynamic (EHD) electroconvection between two plates", J.Phys. D: Appl. Phys, Vol. 41, 175303, 2008.

[23] E. J. Hopfinger and J. P. Gosse, "Charge transport by self-generated turbulence in insulating liquids submitted to unipolar injection", Phys. of Fluids, Vol.14, pp. 1671-1682, 1971.

[24] A. Castellanos, "Basic concepts and equations in electrohydrodynamics", Electrohydrodynamics, 1st ed., Castellanos, A. (ed.), CISM Courses and Lectures No. 380, Springer-Verlag, Berlin, Germany, pp. 1-82,1998.

[25] J. Shrimpton, Charge Injection Systems, Springer-Verlag, Berlin, 2009.

[26] A. Kourmatzis and J.S. Shrimpton, "Electrohydrodynamics and charge injection atomizers: a review of the governing equations and turbulence", Atomization and Sprays, Vol. 19, pp.1045-1063, 2009.

[27] J.S. Shrimpton, "Pulsed charged sprays: applications to DISI engines during early injection", Intern. J. Numer. Meth. Engng, Vol. 58, pp.513-536, 2003.

[28] J.S. Shrimpton and Y. Laoonual, "Dynamics of electrically charged transient evaporating evaporating sprays", Intern. J. Numer. Meth. Engng, Vol. 67, pp. 1063-1081.

[29] G. Malkawi, Point-to-Plane and Plane-to-Plane Electrostatic Charge Injection Atomization for Insulating Liquids, Ph.D. thesis, University of Illinois at Chicago, Chicago IL, USA, 2010.

[30] H.K. Versteeg and W. Malalasekera, An Introduction to Computational Fluid Dynamics: The Finite Volume Method, Longman Scientific \& Technical, 1995

[31] R. Verzicco \& R. Camussi, "Numerical experiments on strongly turbulent thermal convection in a slender cylindrical cell", J. Fluid Mech. Vol. 477, pp. 19-49. 2003.

[32] P.K Yeung, S.B. Pope, B.L. Sawford, "Reynolds number dependence of Lagrangian statistics in large numerical simulations of isotropic turbulence", J. Turbulence. Vol. 7, No.58, 2006.

[33] P.K. Yeung, S.B. Pope, A.G. Lamorgese, D.A. Donzis, "Acceleration and dissipation statistics of numerically simulated isotropic turbulence", Phys. of Fluids, Vol. 18, 065103, 2006.

[34] S. J. Scott, A. U. Karnik, J. S. Shrimpton, "On the quantification of preferential accumulation”, Int. J. Heat and Fl. Flow, Vol. 30, pp. 789-795, 2009

John Shrimpton was born in Essex, England in 1968. He received the B.Eng. degree from Birmingham University, UK in 1992 and the Ph.D. degree from the University of Manchester in 1995. He was appointed a lecturer at Imperial College London, UK in 1998 and was awarded an EPSRC advanced fellowship in 2003. In 2007 and 2010 he was appointed to a senior lectureship and then reader at the University of Southampton, UK.

Agissilaos Kourmatzis was born in Athens, Greece in 1985. He received the B.Eng. degree from the University of Nottingham, UK in 2006 and the M.Sc. degree from Imperial College London in 2007. After a 6 month placement in industry he returned to university and is currently studying for a Ph.D. degree in the electrohydrodynamics of charge injection atomizers at the University of Southampton, UK. 